\title{
Une nanoantenne modulable pour le contrôle de l'amplification du champ optique à l'échelle atomique ${ }^{[1]}$
}

Damien RIEDEL

Institut des Sciences Moléculaires d'Orsay Université Paris Sud, Bâtiment 210

91400 ORSAY, France

damien.riedel@u-psud.fr
$\mathbf{L}^{\mathrm{a}}$ a recherche en optique et plus précisément dans le domaine de la nanooptique a subi un développement considérable depuis ces dix dernières années. De cet essor est né un intérêt commun pour le développement de sources optiques de plus en plus petites et le contrôle de la lumière à des échelles fortement sub-longeur d'onde. Le concept de nanoantenne a ainsi pu voir le jour lorsque la fabrication de nano-barreaux métalliques sur des substrats diélectriques a pu être contrôlée de manière précise [2]. On utilise alors la géométrie de ces lignes (ou plots) et leur espacement précis pour confiner la lumière ou la diriger dans une direction privilégiée [3]. Ces appareils ouvrent ainsi la voie vers de nouveaux moyens pour manipuler la lumière à l'échelle du nanomonde.

Cependant, ces nanoantennes utilisent, dans la majorité des cas, des structures métalliques rigides sans ajustement possible de leurs paramètres, c'est-à-dire sans pouvoir modifier la largeur du nanofil métallique qui la compose ou la distance qui les sépare.

En nanosciences, le besoin de contrôler la lumière aux échelles sub-longueur d'ondes reste un enjeu crucial car ayant un fort impact sur des applications très diverses telles que le ciblage des cellules cancéreuses afin d'aider à leur destruction [4], la photochimie des surfaces (les nez électroniques) permettant la détection de traces de polluants [5], ou même dans l'élaboration des nouvelles géné- rations de cellules solaires dont l'efficacité ne cesse de s'accroître [6].

Pour analyser les prémices de ces processus optiques et agir sur eux à l'échelle du nanomonde, plusieurs outils s'offrent aux ingénieurs et aux chercheurs. Il s'agit des microscopes dits à sondes locales. Ces microscopes utilisent une pointe formée d'une extrémité métallique très effilée surplombant la surface à analyser. Suivant le mode de fonctionnement du microscope (force atomique, optique champ proche, effet tunnel, etc.), divers phénomènes physiques entrent en action pour analyser la topographie et les propriétés de la structure étudiée. Chaque mode de fonctionnement apporte des analyses très spécifiques et souvent complémentaires: le microscope utilisant le champ proche optique permet de mesurer la distribution du champ électromagnétique au voisinage d'un fluorochrome par exemple mais souffre souvent d'une plus faible résolution latérale $(\sim 50 \mathrm{~nm})$. Le microscope à force atomique permet l'observation de larges zones de substrats isolants. Du fait qu'il cartographie les forces à longue portée (de type van der Waals) il possède lui aussi une résolution latérale limitée $(\sim 10 \mathrm{~nm})$. Le microscope à effet tunnel mesure la répartition des densités de charge électronique des surfaces conductrices (métalliques ou semiconductrices) et peut atteindre, dans le meilleur des cas, une résolution latérale de l'ordre de 100 picomètres.

Pour observer et analyser les processus de l'optique en champ proche, les physiciens cherchent à optimiser la capacité de leurs appareils de mesure afin de topographier et de contrôler la répartition du champ électromagnétique à des échelles proches des structures métal- liques dont nous avons parlé précédemment (10-20 nm). Ainsi, en mode de détection, les microscopes à sonde locale dits "sans ouverture ${ }^{1}$ offrent la possibilité de combiner leur mode de fonctionnement propre (le plus souvent les microscopes à force atomique) à la détection de lumière en champ lointain. La pointe du microscope sert alors d'antenne émettrice de la lumière confinée vers le champ lointain grâce à un système de collection de luminescence. Cependant, ces méthodes sont souvent vite limitées par leurs résolutions spatiales et spectrales car elles sont fortement dépendantes de la forme et du matériau constituant la pointe du microscope.

Lorsque l'on s'intéresse au processus inverse, c'est-à-dire lorsque l'on cherche à contrôler le confinement et l'amplification du champ optique dans une nanostructure, depuis le champ lointain vers le champ proche, d'autres problématiques viennent se greffer à celles précédemment citées : (i) comme évoqué précédemment, il est difficile de fabriquer des nanostructures permettant le confinement de champ optique tout en étant capables d'être suffisamment versatiles pour permettre la modulation fine de leurs propriétés, (ii) divers effets photoinduits comme le chauffage thermique de la structure, le photovoltage ou l'émission de photoélectrons peuvent engendrer des difficultés à contrôler et analyser les processus de confinement et d'amplification optique.

Pour remédier à ces problèmes, nous avons utilisé la pointe d'un microscope à effet tunnel (STM) fonctionnant sous

\footnotetext{
"lls sont dits " apertureless» en opposition aux fibres optiques utilisées dans les SNOMs (Scanning Near-field Optical Microscope).
} 
ultra vide et à basse température $(5 \mathrm{~K})$ afin de confiner et amplifier le champ électromagnétique d'un laser pulsé venant irradier la jonction du STM ${ }^{2}$.

La surface observée est une surface de silicium (100) qui a été préalablement passivée par une monocouche d'hydrogène atomique. Dans ces conditions, la pointe métallique du STM va jouer le rôle de nanoantenne alors que la distance $d$ séparant l'apex de la pointe de la surface peut être très précisément ajustée. Nous verrons que ce paramètre d'ajustement est crucial pour nos expériences et l'utilisation de la basse température apporte ici la stabilité nécessaire à son contrôle. Ainsi, le processus d'amplification du champ électromagnétique peut être étudié en fonction de $d$. Par ailleurs, grâce au processus de passivation de la surface, nous avons pu la rendre photosensible et ainsi cartographier la géométrie de la zone d'amplification du champ optique. En effet, la longueur d'onde du laser utilisé (157 $\mathrm{nm}$ ) a été choisie afin d'exciter de manière résonnante la transition antiliante de la liaison Si-H à la surface du silicium. L'excitation de cette transition provoque la désorption des atomes d'hydrogène aux endroits précis où l'intensité du champ optique confiné atteint un certain seuil.

Nous avons réalisé différentes topographies de la surface du Si(100):H à $5 \mathrm{~K}$ avant et après l'irradiation de la jonction du STM par 3400 tirs laser (dose d'irradiation constante, chaque tir laser ayant une durée d'impulsion de l'ordre de 10 ns), et ce, pour différentes hauteurs relatives de la pointe du STM. Dans nos expériences, l'illumination de la jonction est asymétrique comme indiquée sur la figure 1a. Après chaque irradiation, une topographie STM est réalisée (figures $1 \mathrm{~b}$ et $1 c$ ). Sur chacune d'elle, on peut observer une tache de désorption résultante de l'exposition très locale de la surface au rayonnement UV.

\footnotetext{
${ }^{2}$ On parle de jonction STM par analogie aux jonctions Métal-Isolant-Semiconducteur (MIS) développées en électronique.
}

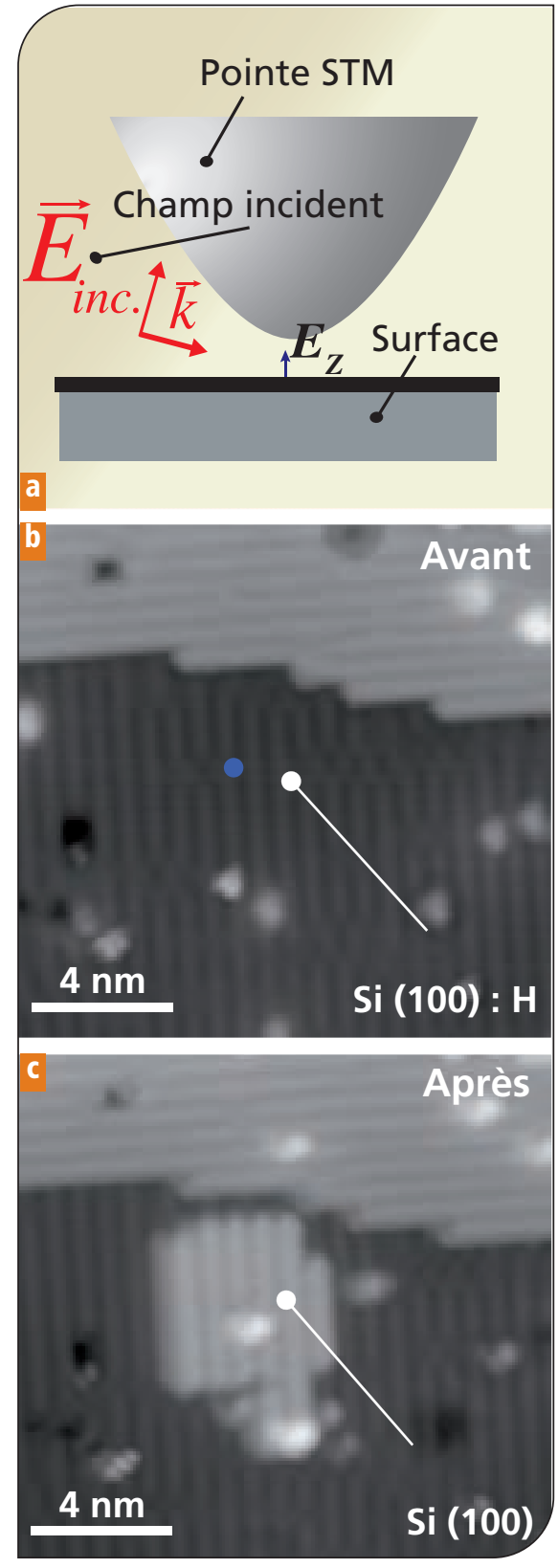

Figure 1a. Schéma du mode d'irradiation (asymétrique) de la jonction du STM. Topographies STM (16 x $\left.16 \mathrm{~nm}^{2}\right)$ illustrant la surface du silicium hydrogénée avant (b) et après (c) l'irradiation laser.

Cette zone de désorption apparaît plus claire sur la topographie STM, car elle correspond à la surface nue du silicium (sans atomes d'hydrogène) via l'imagerie de ses liaisons pendantes (figure 2a).

Comme le montre la figure $2 b$, la tache de désorption observée est le plus souvent de forme elliptique dont le grand axe est toujours orienté selon la direction de propagation du faisceau laser incident lorsque celui-ci est polarisé de manière rectiligne (figure 1a).

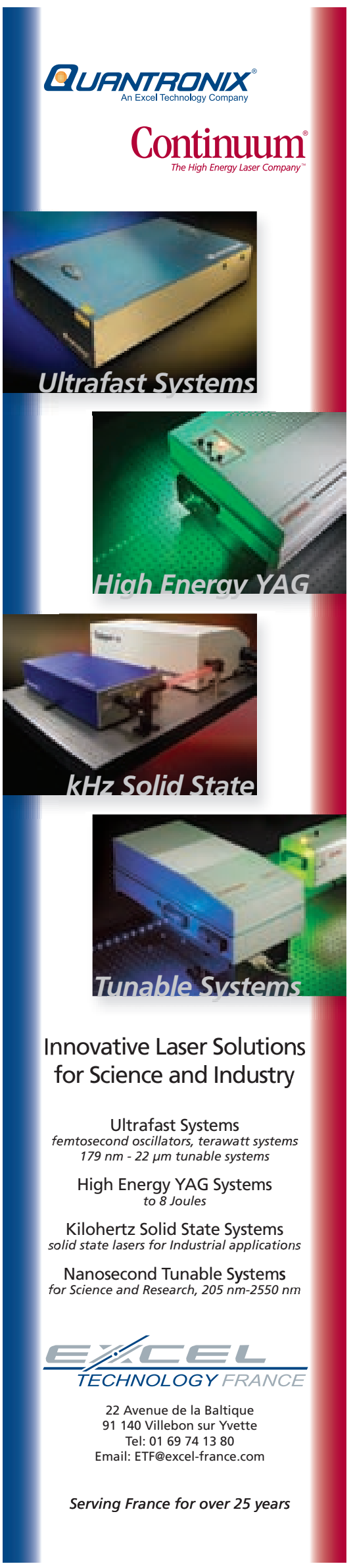



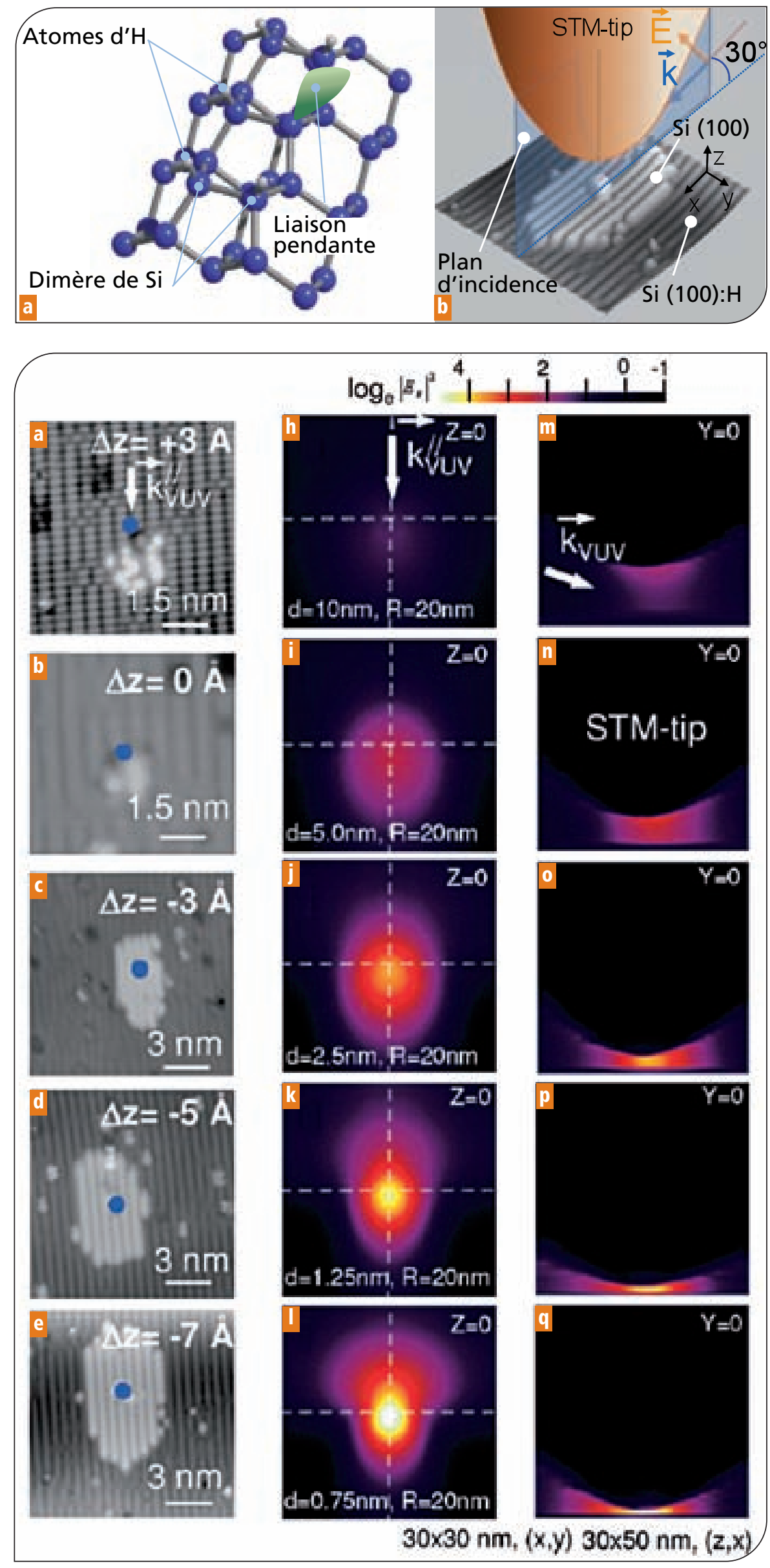

Figure 2a. Vue synthétique partielle de la surface du silicium (atomes en bleu) hydrogénée (atome en blanc) avec une liaison pendante (orbitale verte). b. Positions relatives de la pointe du STM avec la surface et les différentes composantes du champ électromagnétique ainsi que la direction de l'irradiation laser par rapport à la zone déshydrogénée créée.

La taille de la tache de désorption est d'autant plus grande que la distance pointe-surface est faible (figures $3 a$ à $3 e$ ). On note également un léger décalage entre la position du centre de l'ellipse et la position de l'apex de la pointe du STM (matérialisé par des points bleus). Pour pouvoir observer ce phénomène de désorption de l'hydrogène très localisé, il est nécessaire de maîtriser de manière très précise et répétable la distance pointe-surface, qui en totalité ne varie ici que de 10 angströms. Ceci est possible grâce à l'utilisation du STM à basse température. Il est également important de noter que ces expériences se font sans électrons tunnels pendant l'irradiation ${ }^{3}$ permettant ainsi d'attribuer nos résultats au seul confinement optique et à l'amplification du champ UV à l'apex de la pointe du STM.

En calculant la distribution de la composante verticale du champ électrique à la surface du silicium (figure 3), nous avons pu montrer que la taille de la tache de désorption correspondait très précisément à la cartographie expérimentale obtenue de la distribution du champ

\footnotetext{
${ }^{3}$ Pendant l'irradiation, la boucle de rétroaction du microscope qui sert au mode de fonctionnement en courant constant est stoppée et redémarrée à la fin de l'irradiation. Durant cette période, la tension appliquée à la surface est fixée à 0 volt pour revenir ensuite à la tension d'imagerie.
}

Figure 3. De (a) à (e), série de topographies STM montrant différentes zones de désorption correspondant au champ optique confiné pour différentes hauteurs relatives $\Delta z$ de la pointe du STM. Les distributions calculées du champ électrique (composante verticale) dans le plan de la surface du silicium ( $h$ à $/$ ) et dans le plan perpendiculaire à la surface du silicium ( $m$ à $q)$ sont représentées en fonction de diverses distances pointe-surface $d$. 


\section{CRHIER TECHNIDUE 53}

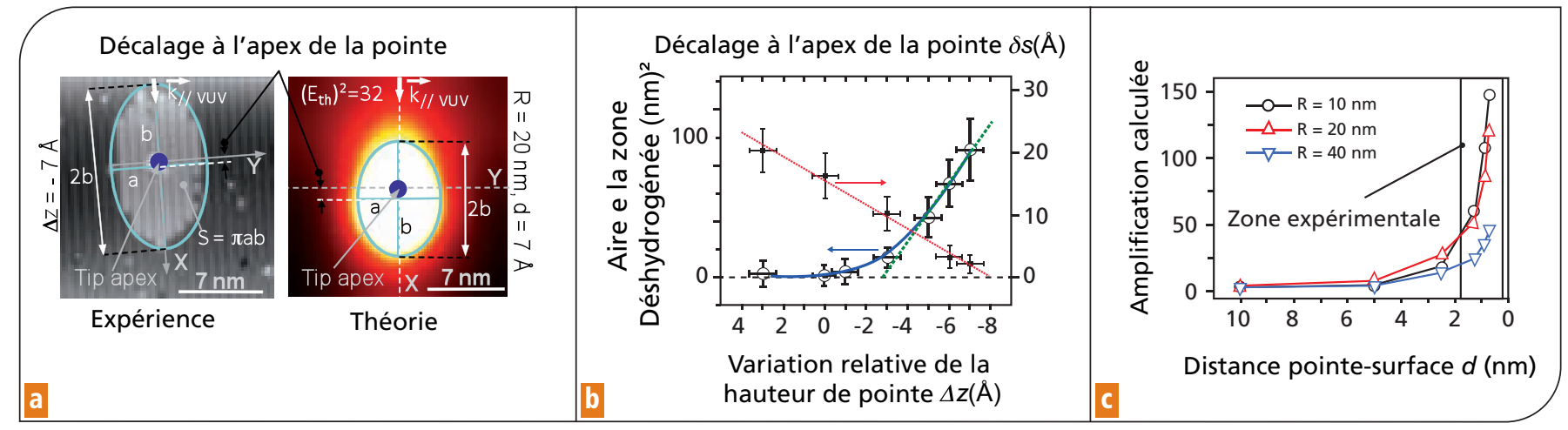

Figure 4a. Comparaison entre la topographie STM de la tache de désorption expérimentale (gauche) et le «hot spot » optique calculé (droite) pour une pointe de rayon de courbure $20 \mathrm{~nm}$ et une distance pointe-surface de 7 angströms. b. Variation moyenne de la taille de la zone de désorption expérimentale et du décalage à l'apex en fonction de la hauteur relative pointe-surface pour différentes pointes STM. c. Variation de l'amplification calculée en fonction de la distance pointe-surface $d$.

électrique alors que celle-ci augmente progressivement en taille en approchant la pointe de la surface. En effet, comme le montrent les figures $3 h$ à $3 q$, lorsque la pointe est relativement éloignée de la surface $(d \sim 10 \mathrm{~nm})$, le champ optique est principalement confiné à l'apex de la pointe du STM avec une faible amplification $(\rho \sim 8)$. Au fur et à mesure que la pointe du STM se rapproche de la surface du silicium, l'amplification et le confinement augmentent à la surface du silicium ( $\rho \sim 150)$ permettant d'agir photochimiquement sur celle-ci. Ainsi, grâce à cette méthode, il est possible d'appliquer à la surface d'un matériau un champ électromagnétique très confiné dont l'intensité ainsi que la distribution spatiale peuvent être finement contrôlées.

Il est important de noter que grâce à l'utilisation combinée du STM à basse température avec une longueur d'onde UV, les effets photothermiques qui sont souvent une limite majeur à ce type d'expérience sont ici négligeables [7], et permettent l'ajustement très précis de I'amplification du champ optique sous la pointe du STM. Comme nous l'avons observé expérimentalement et théoriquement, la distribution du champ optique confiné est anisotrope et de forme elliptique (figure 4a). L'origine de cette anisotropie ne provient pas de la forme de l'apex de la pointe du STM. En effet, notre méthode de gravure des pointes STM utilisées impose une longueur de pointe précise impliquant une bonne répétabilité de leurs rayons de courbure et, de là, une grande efficacité de confinement dans la plupart des cas. Nous avons en revanche pu montrer que la forme elliptique de la distribution du champ optique est à l'origine d'un très fort couplage entre le dipôle induit dans la pointe du STM et le dipôle image créé à la surface du silicium par le champ UV car, à la longueur d'onde utilisée, le silicium agit comme un métal [1].

En réitérant l'opération de cartographie de la répartition du champ optique amplifié avec différentes pointes STM, nous avons observé que la taille de la zone déshydrogénée augmente linéairement lorsque la pointe se rapproche de la surface (figure 4b). Inversement, le décalage entre la position de l'apex de la pointe et le centre de la tache de désorption diminue lorsque la distance relative entre la pointe et la surface se réduit. Nos calculs reproduisent ces variations et indiquent une rapide modification de l'amplification du champ optique confiné lorsque la distance $d$ diminue (figure 4c). De plus, en reproduisant la tache de désorption expérimentale ( $f i$ gure 4a), nos expériences montrent que la zone déshydrogénée peut atteindre des tailles extrêmement petites ( $6 \mathrm{~nm})$ repoussant ainsi les limites de diffraction et les possibilités de contrôle du confinement optique.

Ce travail original représente une avancée considérable en nanophotonique et plus particulièrement dans le contrôle des champs optiques confinés car il ouvre la voie vers de nouvelles expériences scientifiques visant, par exemple, au développement de microscope de nouvelle génération permettant d'avoir des accès de mesure ou d'excitation multipoints. Cette recherche possède également un impact évident dans le domaine de la lithogravure photochimique à l'échelle atomique permettant $d^{\prime}$ 'imaginer des méthodes efficaces et précises de gravure de couches minces ou de corrections de circuits intégrés. Dans ce cadre, I'utilisation d'appareils fonctionnant à basse température peut sans doute devenir très commune car ils offrent de multiples avantages au contrôle des processus nanophotoniques. Enfin, ces travaux ouvrent également de nouvelles perspectives en vue de combiner des champs optiques façonnés plus complexes sur des structures moléculaires individuelles fonctionnalisées afin de les activer optiquement de manière très spécifique.

\section{Références}

[1]D. Riedel, R. Delattre,A.G. Borisov, T.V.Teperik. Nano Lett. 10, pp. 3857-3862 (2010).

[2] S. Kawata etal., Nat. Photon. 3, pp. 388-394 (2009).

[3] A. G. Curto et al., Science 329, pp. 930-933 (2010).

[4] Rizia Bardhan et al., Nano Lett. 10, pp. 4920-4928 (2010).

[5] Y. Chang-Cheng, Nat. Nanotechnol. 2, pp. 318-323 (2007).

[6] J. B. Sambur et al., Science 330, pp. 63-66 (2010). [7] D. Riedel et al., Phys. Rev. B 80, pp. 155451-155457 (2009). 